\title{
Processing of Non-Impulsive Moving Sources data for structural and 4D imaging: illustrations on SEAM models
}

\author{
Antoine Guitton, Bertrand Duquet, Stephen Secker, Jean-Patrick Mascomere, Andrew Feltham, Total E\&P, Pau, France.
}

Copyright 2021, SBGf - Sociedade Brasileira de Geofísica

This paper was prepared for presentation during the $17^{\text {th }}$ International Congress of the Brazilian Geophysical Society held in Rio de Janeiro, Brazil, 16-19 August 2021.

Contents of this paper were reviewed by the Technical Committee of the $17^{\text {th }}$ International Congress of the Brazilian Geophysical Society and do not necessarily represent any position of the SBGf, its officers or members. Electronic reproduction o storage of any part of this paper for commercial purposes without the written consent of the Brazilian Geophysical Society is prohibited.

\begin{abstract}
Marine vibrator sources offer many advantages for seismic acquisitions compared to classical airguns (Laws et al, 2019). The first advantage is a reduced environmental footprint, emitting only the required frequencies together with a significant reduction of the instantaneous peak energy due to an increased emission time. It can also emit very repeatable and specially tailored signals to favor specific frequency bands. One specificity of marine vibrators is that they emit for few seconds while the source vessel is in motion, which results in recorded data affected by the Doppler effect. The Doppler effect is a function of both the boat speed and the apparent velocity of the seismic events. We investigate pre-processing steps to mitigate the Doppler effect and analyze its impact for 3D and 4D imaging.
\end{abstract}

\section{Introduction}

Several authors (Dragoset, 1988; Hampson and Jakubowicz 1995; Qi and Hilterman, 2016; Asgedom et al., 2019) have studied the Doppler effect associated with nonimpulsive moving sources (NIMS) and proposed methods to correct for it. In this paper we consider a 2D OBN acquisition geometry where only the source displacement must be accounted for. In this case, as described by (Hampson and Jakubowicz, 1995), the NIMS effect can be corrected by cross-correlating the common-receiver data with the moving source, which becomes a 2D function. In the $(\omega, k)$ domain such cross-correlation can be written as

$$
d_{x_{r}}\left(\omega, k_{x_{s}}\right) \overline{S\left(\omega-k_{x_{s}} V_{b}\right)},
$$

where the $d_{x_{r}}\left(\omega, k_{x_{s}}\right)$ represent the recorded marine vibrator data sorted into common-receiver gathers (CRG) in the Fourier domain, $S(\omega)$ is the sweep signature and $V_{b}$ the boat velocity. When $V_{b}=0 \mathrm{~m} / \mathrm{s}$ in equation 1 , we recognize the simple cross-correlation of the data with the sweep signature, as applied classically in land processing for acquisitions using vibrator trucks.

For marine vibrator acquisitions, the cross-correlation in equation 1 involves the boat velocity and the underlying source sampling, which is the distance between two consecutive sweep emissions. In this paper we consider, as specified in Feltham et al. (2018), a linear sweep duration of $5 \mathrm{~s}$ and a boat velocity of $4.8 \mathrm{~m} / \mathrm{s}$, thus yielding a linear sweep emission every $24 \mathrm{~m}$. At first glance, a 4.8 $\mathrm{m} / \mathrm{s}$ (9.3 knots) boat velocity seems high, considering most towed streamer seismic acquisition vessels travel at approximately $2.3 \mathrm{~m} / \mathrm{s}$ (4.6 knots) water velocity. If we consider the impact of ocean currents, which can reach up to $2.5 \mathrm{~m} / \mathrm{s}$ in places like Brazil (Duncan et Schladow, 1981), $4.8 \mathrm{~m} / \mathrm{s}$ becomes a reasonable case that we must investigate. Furthermore, understanding the impact of the Doppler effect at $4.8 \mathrm{~m} / \mathrm{s}$ (9 knots) might enable faster seismic acquisitions and, as a result, improve the efficiency and reduce the cost of seismic surveys.

As described in Dragoset (1988), the Doppler effect due to the source motion is proportional to

$$
\mathrm{V}_{\mathrm{b}}{ }^{*} \mathrm{p}_{\mathrm{x}_{\mathrm{s}}}
$$

where $p_{x_{s}}=k_{x_{s}} / \omega$ is the apparent slope of the recorded events in the CRGs. The cross-correlation in equation 1 will correct for this doppler effect but may suffer from aliasing artefacts due to the shot sampling, as we will illustrate below.

\section{Seismic Modeling and validation of NIMS correction}

In order to study the impact of the Doppler effect and its mitigation, we need to properly model NIMS data numerically. To do so, two aspects must be accounted for: first, a precise propagation of the sweep up to $100 \mathrm{~Hz}$ to fulfill the specifications described in Feltham et al. (2018) and second, a correct injection of the moving sweep in the Finite Difference (FD) numerical grid. For the later, we use a simple linear interpolation and calibrate our modeling by comparing the direct arrival in a homogeneous medium $(\mathrm{v}=1500 \mathrm{~m} / \mathrm{s})$ with an analytical solution. For our FD propagator ( $2^{\text {nd }}$ order in time and $4^{\text {th }}$ order in space), a spatial grid sampling of $1 \mathrm{~m}$ and a time sampling of $0.1 \mathrm{~ms}$ provide results without visible distortions, as illustrated in Figure 1. Such a fine sampling is tractable in 2D but other strategies will be needed in fo 3D.

Using this simple homogeneous case, we model a CRG for a stationary and a moving sweep. We modeled first a nonrealistic case of a sweep emission every $6 \mathrm{~m}$ (equivalent to an experiment where the boat would be required to shoot the same line four times), ensuring that the direct arrival remains unaliased for the full frequency band. In Figure $2 a$, we compare the CRGs associated with a stationary source and cross-correlated with the sweep (equation 1 using $V_{b}=$ 0 ), in Figure $2 b$ the result of the common receiver associated with a moving source emitting a sweep every 6 $\mathrm{m}$ or every $24 \mathrm{~m}$ (Figure $2 \mathrm{c}$ ). Note that in both Figures $2 \mathrm{~b}$ and $2 \mathrm{c}$, the CRGs are crosscorrelated using equation 1. 


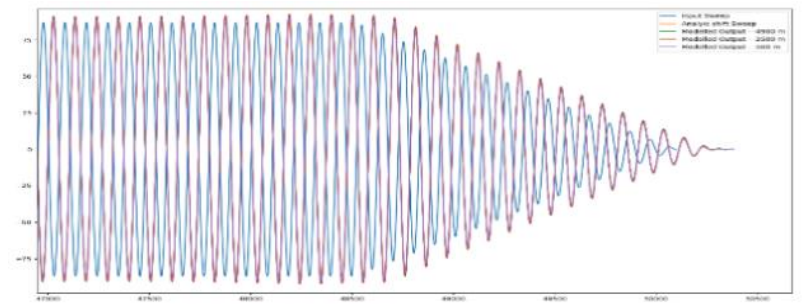

Figure 1: Display of the input sweep, analytically doppler shifted sweep and direct arrival modeling result for different offsets. We consider here a case of source and receivers placed at the same depth and each modelled trace is time shifted by the associated travel time from source to receiver. We display the tail of the sweep that should exhibits the most dispersion. As can be observed, all modelled traces exhibit the same doppler shift and match well the analytically doppler shifted one.

Figure $2 d$ displays two traces for these CRGs and shows an excellent match between Figures $2 \mathrm{a}$ and $2 \mathrm{~b}$ (in blue) and discrepancies with Figure 2c. Any Doppler correction applied to Figure $2 \mathrm{c}$ should make it look like Figure $2 \mathrm{a}$. The difference stems from the coarse shot sampling $(24 \mathrm{~m})$ and the fast-moving boat $(\mathrm{Vb}=4.8 \mathrm{~m} / \mathrm{s})$ when the Doppler effect is present, thus resulting in aliasing artefacts.

\section{Deconvolution/interpolation for aliasing mitigation}

To remedy the aliasing issue, we propose an inversion framework that simultaneously deconvolve the sweep and reconstruct traces at an optimal shot spacing, effectively adding more shot points, thus mitigating the aliasing artifacts. This approach introduces a composite linear operator that includes a mapping operator between the true and reconstructed shot positions, a multidimensional convolution operator to simulate the moving sweep, and a dictionary that maps the seismic data into a basis function where the sparseness of the signal is utilized to recreate the missing shots. This approach achieves three important goals: the data are deconvolved of the moving sweep function, free of aliasing artifacts, to a shot sampling that can be later used for other processing steps. We also propose an extension of our approach to process blended shots.

Assuming an ideal acquisition setup where the boat moves at a constant speed while emitting a repeatable sweep signal, with a non-varying sea surface, the relationship between an observed common-receiver gather $\mathbf{d}$ from a moving sweep signal and its deconvolved counterparts $\mathbf{d}_{i}$ becomes (Hampson and Jakubowicz, 1995)

$$
\mathbf{C d}_{\mathbf{i}} \stackrel{\text { def }}{=} \mathbf{d}
$$

where $\mathbf{C}$ is a slanted, multi-dimensional stationary convolution operator with the sweep (2D or $3 D$ ). This convolution operator depends on the spatial (i.e. shot) sampling in the receiver gather which, in turn, depends on the boat speed and on the sweep signal. For a non-moving source, the convolution operator becomes $1 \mathrm{D}$ and no Doppler effect is present. We call $\mathbf{C}_{1 \mathrm{D}}$ the convolution operator with the 1D sweep. As shown by Qi and Hilterman (2016), the simple correlation of the 1D sweep with the recorded NIMS data generates phase errors, due to the Doppler effect, that need to corrected for. In theory, the correlation of the 2D sweep with the recorded data should yield phase-corrected traces. However, due to the shot sampling in the CRGs, the 2D correlation will also generate aliasing artifacts. We propose an approach that handles the aliasing artifacts while deconvolving the CRGs. The key idea is to interpolate the missing traces (shots) to a finer grid to mitigate the aliasing artifacts by solving the following basis-pursuit denoise problem:

$$
\min |\mathbf{m}|_{1} \text { s.t. }\|\mathbf{S C L m}-\mathbf{d}\|_{2}^{2} \leq \sigma
$$

where $\mathbf{L}$ is the linear Radon transform (LRT) on the finer grid, $\mathbf{S}$ is a mapping operator between the acquisition and reconstructed grids, $||$.1 is the $\ell 1$ norm that enforces sparseness in the Radon domain, and $\sigma$ is the noise level expected in the data. In practice, $\sigma$ is selected as a function of the data RMS. In this scheme, the convolution happens on well sampled data $\mathbf{L m}$, which are then mapped to the acquisition grid. Therefore, $\mathbf{S}$ can be a subsampling, masking, or interpolation operator depending on the acquisition geometry. In practice, the data $\mathbf{d}$ is first decomposed into patches and each patch is processed independently with equation 2, thus allowing fast processing on an HPC environment. Notice that we can use other transforms as well, such as curvelets, for the interpolation step. If curvelets are used, compressive sensing principles should be incorporated at the acquisition stage to get the best possible uplift. Also, our approach works for any sweep function. We illustrate this approach on a 2D synthetic dataset modeled from a scaled version of the SEAM Phase 1 model.

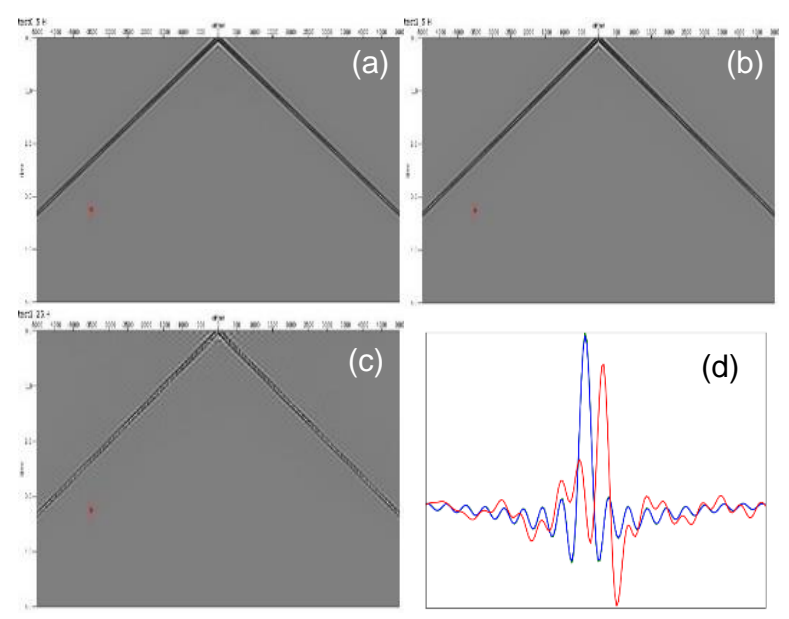

Figure 2: Direct arrival modeled in a homogeneous medium ( $v=1500 \mathrm{~m} / \mathrm{s})$ for (a) a stationary source correlated with the sweep, a moving source emitting every (b) $6 \mathrm{~m}$ and (c) $24 \mathrm{~m}$ and cross-correlated using equation 1. (d) shows a zoom (between $2.2 \mathrm{~s}$ and $2.4 \mathrm{~s}$ ) of an extracted trace at offset $3500 \mathrm{~m}$ for the three results: the results for (a) and (b) (in blue) are the the same, while the trace in red for (c) highlights the Doppler effect for a fast moving sourcen $(\mathrm{Vb}=4.8 \mathrm{~m} / \mathrm{s})$ with a coarse shot sampling of $24 \mathrm{~m}$.

We first apply our deconvolution-interpolation approach to the non-moving sweep data, replacing $C$ by $C 1 D$ in equation 4. Figure 3a shows a small window where events with conflicting dips from different horizons (direct arrivals 
and salt reflections) meet: these events experience different Doppler effects due to their apparent dips. Therefore, some collocated reflections experience an increase or decrease of their frequency content. Without Doppler effect, and spatial aliasing, Figure 3b displays the deconvolution result SLm where $\mathbf{m}$ is the estimated Radon model (in patches): we consider this result as the "ideal" answer of our inversion with the moving sweep that we are trying to match. In all our results, unless otherwise indicated, $\mathbf{S}$ is a subsampling operator that takes the data from a $24 \mathrm{~m}$ grid onto a $6 \mathrm{~m}$ grid.
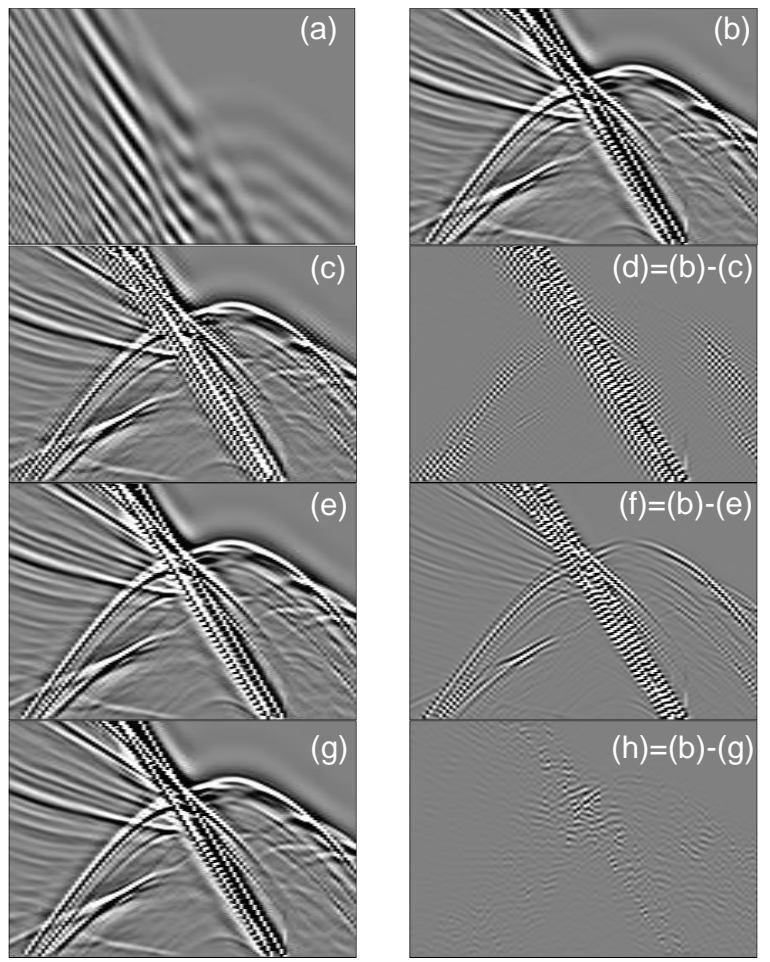

Figure 3: (a) Input data modeled with a linear sweep using our finite difference approach. (b) Deconvolved data without moving sweep: the answer. (c) Deconvolved data with 2D operator without interpolation to $6 \mathrm{~m}$. (d) Difference (c) - (b). (e) Deconvolved data with 1D operator with interpolation to $6 \mathrm{~m}$. (f) Difference (e) - (b). (g) Deconvolved data with 2D operator with interpolation to $6 \mathrm{~m}$. (h) Difference (g) - (b). Except for Figure 3a, all subfigures are plotted on the same scale.

Let's now consider the processing of moving NIMS data with Doppler effect. If we ignore the shot sampling issue and no interpolation is done in equation 4 (reducing $\mathbf{S}$ to a simple identity operator), we obtain Figure 3c, where strong aliasing artifacts are present (Figure $3 \mathrm{~d}$ shows the difference between Figure $3 c$ and Figure $3 b$ ). Similar effects have been reported by Qi and Hilterman (2016). Note that the phase of events with the fastest apparent velocities matches those from the "ideal" answer, while it does not for the slowest ones where the Doppler effect is the strongest. If we apply our deconvolution/interpolation approach to the moving sweep data, replacing $C$ by C1D in equation 4, we get Figure $3 e$, where Figure $3 f$ displays the difference with Figure $3 \mathrm{~b}$. The phase errors affect all events (except those with zero apparent velocities) and would need to be corrected for in post-processing. Finally, Figure $3 \mathrm{~g}$ shows the deconvolution result using the 2D convolution operator on a $6 \mathrm{~m}$ grid (equation 4), and Figure $3 \mathrm{~h}$ displays the difference with Figure $3 \mathrm{~b}$ : the aliasing and phase errors seen in the other two results are almost entirely attenuated, thus validating our proposed approach.

\section{Impact of NIMS correction for structural imaging}

We now consider the same 2D line of the scaled SEAM model and illustrate the impact of the NIMS correction on the migrated images. We model data without a free surface for a stationary (the reference) and a moving sweep. For the moving sweep, we also consider the non-realistic case of a sweep emission every $6 \mathrm{~m}$. The data are crosscorrelated and the direct arrival is muted before running a 2D RTM with a smooth version of the exact model. In all cases, we migrate with a shot sampling of $24 \mathrm{~m}$. For the stationary sweep data, we use $V_{b}=0$ for the crosscorrelation, while for the moving sweep we consider two cases:

1. Cross-correlate the data with the sweep $\left(V_{b}=0\right)$ and redefine the source position to be at the center of the sweep emission (lateral shift of 12 $\mathrm{m})$. We will call this the 1D correction.

2. Apply the $2 \mathrm{D}$ cross-correlation (equation 1) with the correct boat velocity. We will call this the 2D correction.

The first case is a 1D approximation that won't suffer from aliasing and will, in average, position the cross-correlated shots at their correct position but won't correct for the doppler effect.

Figures $4 \mathrm{a}, 4 \mathrm{c}, 4 \mathrm{e}$, and $4 \mathrm{~g}$ illustrate the different migrated images while Figures $4 \mathrm{~b}, 4 \mathrm{~d}, 4 \mathrm{f}$, and $4 \mathrm{~h}$ shows the differences with the reference result (stationary sweep data). The non-realistic case of a sweep emission every 6 $\mathrm{m}$, correlated with a $2 \mathrm{D}$ sweep function with $\mathrm{Vb}=4.8 \mathrm{~m} / \mathrm{s}$ gives an excellent imaging result. For a sweep emission every $24 \mathrm{~m}$, both 1D and 2D corrections exhibit differences in the shallow part, with the $2 \mathrm{D}$ correction showing differences that vanish with depth. Indeed, the shallow section is associated with events having strong apparent slopes in the common receiver data that are not high cut filtered by the migration process. For those events at least, the deconvolution/interpolation approach detailed above should be used.

\section{Impact of NIMS correction for 4D imaging}

The 2D correction error is hardly visible for depths greater than $1500 \mathrm{~m}$ (Figure $4 \mathrm{~g}$ ) but can be observed on differences images (Figure 4h). We can therefore wonder about the impact of this correction for 4D analysis.

Therefore, we extract a 2D line of a scaled SEAM 4D model and reduce by a factor three the original 4D differences in order to illustrate the impact of NIMS corrections on weak 4D signal. Figure 5 shows the 4D differences for different acquisition scenarios:

a) Figure 5a: both base and monitor data acquired with stationary sweep (reference result) 
b) Figure 5b: both base and monitor data acquired with moving sweep (1D correction).

c) Figures $5 \mathrm{c}$ and $5 \mathrm{~d}$ : base is acquired with stationary sweep and monitor data acquired with moving sweep using 1D (5c) and 2D (5d) corrections.

When both data are acquired with a moving sweep (Figure $5 b$ ), the $1 \mathrm{D}$ and $2 \mathrm{D}$ corrections provide a very accurate $4 \mathrm{D}$ signal (the 2D correction is not displayed here because it looks very similar to the 1D correction in Figure 5b), almost equivalent to the reference result in Figure $5 \mathrm{a}$. For the more challenging case where the base and monitor surveys are using a static and a moving source, respectively, the $1 \mathrm{D}$ correction exhibit errors on the whole section while those for the 2D correction are strongly attenuated below $1500 \mathrm{~m}$. This can be also observed on normalized RMS (NRMS) difference displays (Figures $5 \mathrm{e}$ to $5 \mathrm{~h}$ ). These figures show the differences between the NRMS calculated for the reference results (both base and monitor surveys use a static sweep) with the NRMS calculated with the previous acquisition scenarios. These differences of NRMS highlight where the Doppler effect, and our corrections, affect the 4D signal the most. On these displays we can observe that the 1D correction in Figure 5e, that doesn't account for the Doppler effect at all $(\mathrm{Vb}=0 \mathrm{~m} / \mathrm{s}+$ averaging of the source location), shows overall small errors that increase with depth and locally error up to $5 \%$ along the fault in the $4 \mathrm{D}$ area, while the $2 \mathrm{D}$ correction provides a small $(<1 \%)$, homogeneous error (Figure 5f). In Figure 5g, where the base is using a static source and the monitor a moving source, the 1D correction clearly displays strong NRMS differences close to $+/-5 \%$. Below $1500 \mathrm{~m}$ the difference of NRMS between the reference results and the $2 \mathrm{D}$ correction is smaller than $1 \%$.

\section{Discussion}

We illustrate, for the case of a linear sweep, the sensitivity to aliasing of the NIMS correction using 2D crosscorrelation. The Doppler effect is a function of the apparent data slope and boat velocity (equation 2). Even though a high boat velocity is used, we illustrate that for deep targets (below $1500 \mathrm{~m}$ depth in our examples) the Doppler correction error is small. For shallower targets, the boat velocity needs to be reduced or a deconvolution/interpolation approach (equation 4) can be used. However, we have considered here a simple modeling approach where ghost and multiples haven't been introduced. We believe an extension including variable sea state (and therefore variable ghosted sweep) should also be considered. Moreover, for better productivity and for reducing the distance between sweeps continuous shooting/emission, together with a deblending approache, need to be considered. It is also our belief that a more advanced modeling exercise for Non-Impulsive Moving Sources including all these different acquisition and processing aspects, should be considered by SEAM as a Joint Industry Project.

\section{Conclusion}

We have illustrated the impact of NIMS corrections for structural and 4D imaging. Although not impacting imaging at reservoir levels, the tested corrections are sensitive to aliasing and exhibit errors in shallow areas. This aliasing is a direct consequence of the Doppler effect, which plagues NIMS data for fast boats and steeply dipping events. We propose a deconvolution/interpolation approach based on a sparse Radon transform to mitigate these effects.

In order to confirm these results, a more complete 3D modeling exercise including variable sea state, ghost and surface-related multiples should be considered to further assess the impact of NIMS to imaging and reservoir characterization.

\section{Acknowledgements}

We thank Total E\&P for permission to publish these results. We also thank A. Lafram, C. Agut and E. Zamboni for fruitful discussions.

\section{References}

Asgedom, E., O. Orji, and W. Sollner, 2019, Marine vibrator sources: Motion correction, deghosting and designature: 81st Conference and Exhibition, EAGE, Extended Abstracts, Th P05 10.

Dragoset, W. H., 1988, Marine vibrators and the doppler effect: Geophysics, 53, 1388-1398.

Duncan, C. P., and Schladow, S.G., 1981, World surface currents from ship's drift observations: International Hydrographic review, Monaco, LVIII (2), July 1981.

Feltham, A., Girard M., Jenkerson, M., Nechayuk, V., Griswold, S., Henderson, N. and Johnson, G., 2018, The Marine Vibrator Joint Industry Project: four years on: Exploration Geophysics, v 45, p 675-687.

Hampson, G., and H. Jakubowicz, 1995, The effects of source and receiver motion on seismic data1: Geophysical Prospecting, 43, 221-244.

Laws, R., D. Halliday, J.-F. Hopperstad, D. Gerez, M. Supawala, A. Ozbek, T. Murray, and E. Kragh, 2019, Marine vibrators: the new phase of seismic exploration: Geophysical Prospecting, 67, 1443-1471.

Qi, C., and F. Hilterman, 2016, Removal of doppler effects from marine vibrator OBN seismic: 86th Annual International Meeting, SEG, Expanded Abstracts, 183187. 

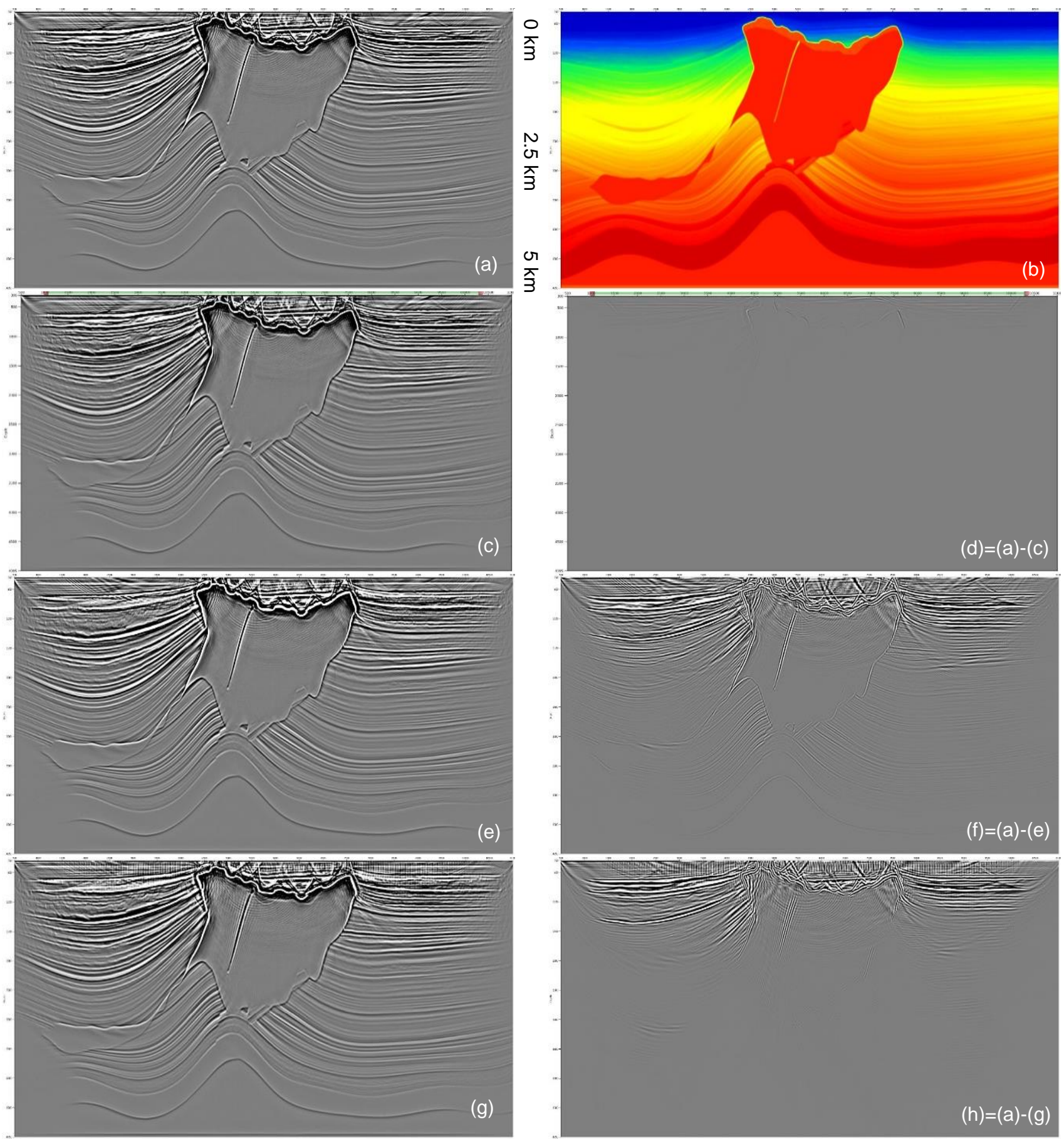

Figure 4: Seam Phase 1 migrated images associated with (a) modeled data with stationary sweep cross-correlated with the sweep (reference result), (c) modeled data with moving sweep emission every $6 \mathrm{~m}$ and 2D correction, (e) modeled data with moving sweep emission every $24 \mathrm{~m}$ and 1D correction, (g) modeled data with moving sweep emission every $24 \mathrm{~m}$ and $2 \mathrm{D}$ correction. (b) Scaled SEAM Phase 1 velocity model 2D extraction used for the modeling. (d) Difference between (a) and (c). (f) Difference between (a) and (e). (h) Difference between (a) and (g). 

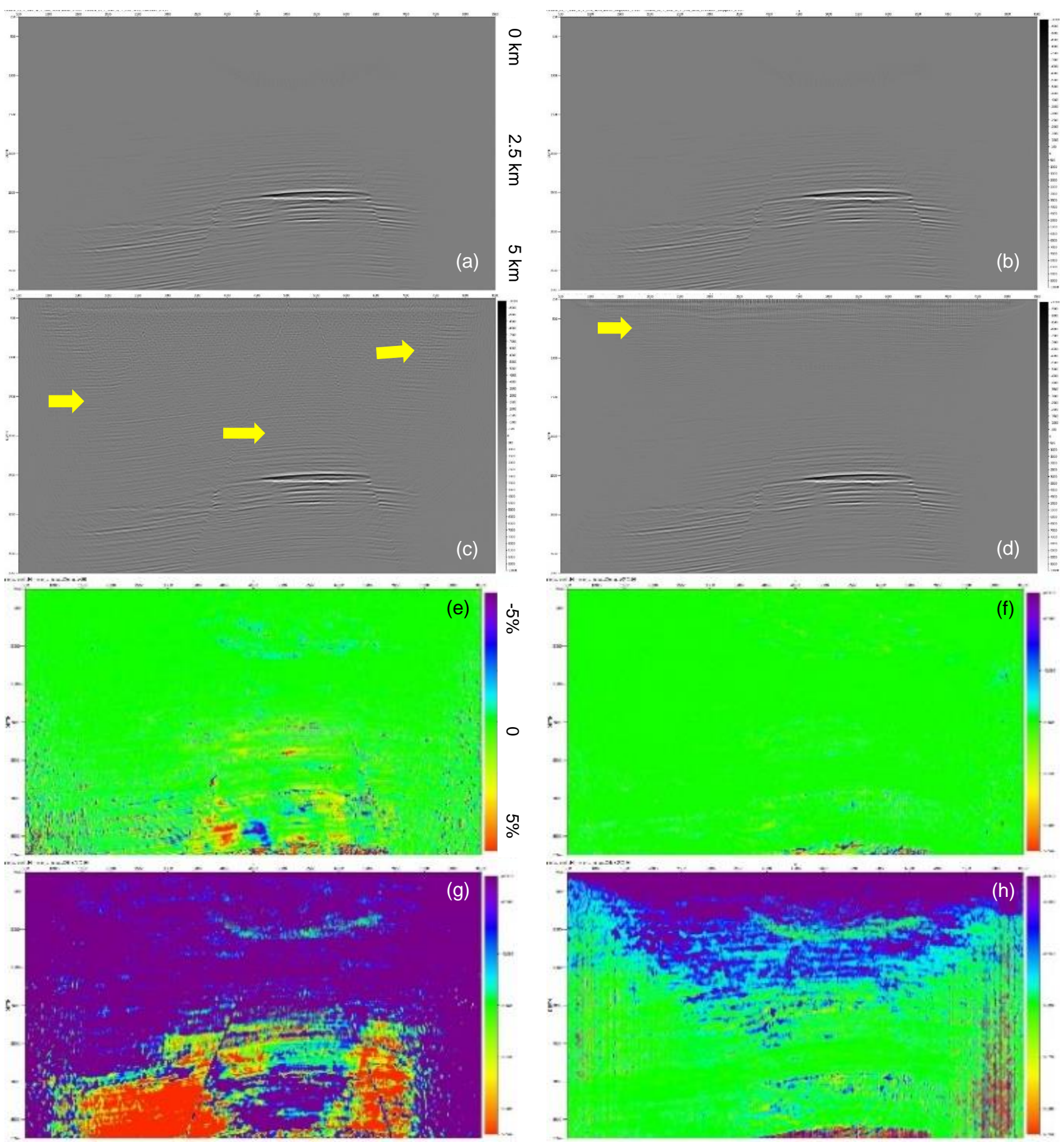

Figure 5: Seam 4D differences in the depth migrated domain for (a) base and monitor data modeled with stationary sweep and cross-correlated with the sweep (reference result), (b) base and monitor data modeled with moving sweep and using 1D correction, (c) base data modeled with stationary sweep (cross-correlated with the sweep) and monitor data modeled with moving sweep and using 1D correction, (d) base modeled with stationary sweep (cross-correlated with the sweep) and monitor data modeled with moving sweep and using 2D correction. NRMS differences between reference NRMS (NRMS of base and monitor differences for data modeled with stationary sweeps) and NRMS obtained with (e) base and monitor differences obtained for moving sweeps with 1D correction, (f) base and monitor differences obtained for moving sweeps and 2D corrections, $(\mathrm{g})$ difference between base obtained with stationary sweeps and monitor obtained with moving sweeps with 1D correction, (h) difference between base obtained with stationary sweeps and monitor obtained with moving sweeps with 2D correction. The color map ranges from $-5 \%$ (blue) to $5 \%$ (red). Green color indicates a difference of NRMS smaller than $1 \%$. 